\title{
Radio sources in the Massive and Distant Clusters of the WISE Survey (MaDCoWS)
}

\section{Ruta Kale ${ }^{* a}$ and Viral Parekh ${ }^{b}$}

${ }^{a}$ National Centre for Radio Astrophysics, Tata Institute of Fundamental Research, Post Bag 3, Pune University Campus, Pune 411007, India

${ }^{b}$ Raman Research Institute, C. V. Raman Avenue, Sadashivnagar, Bangalore 560080, India

E-mail: ruta@ncra.tifr.res.in, viralerri.res.in

Radio sources in galaxy clusters at high redshifts $(z>0.8)$ remain less explored due to the limitations of existing radio surveys and the dearth of known clusters at those redshifts. Recently new high redshift $(\sim 1)$ clusters have been detected using the Wide-field Infra-red Survey Explorer (WISE). We present preliminary results from our radio survey of the Massive and Distant Clusters from the WISE Survey (MaDCoWS) with the Giant Metrewave Radio Telescope.

EXTRA-RADSUR2015 (*)

20-23 October 2015

Bologna, Italy

(*) This conference has been organized with the support of the Ministry of Foreign Affairs and International Cooperation, Directorate General for the Country Promotion (Bilateral Grant Agreement ZA14GR02 - Mapping the Universe on the Pathway to SKA)

\footnotetext{
* Speaker.
} 


\section{Introduction}

Radio surveys of clusters of galaxies are necessary to study the occurrence and evolution of AGN in the dense environments of the intra-cluster medium (ICM). All sky radio surveys with sufficient depth have already facilitated studies of nearby clusters but have limitations to study high redshift clusters due to insufficient resolution and sensitivity. For example, the widely used all sky surveys such as the NRAO VLA Sky Survey (NVSS,[1]), the Sydney University Molonglo Sky Survey (SUMSS, [2]), VLA Low Frequency Sky Survey (VLSS, [3]), the Westerbork Northern Sky Survey (WENSS, [4]) have resolutions in the range $45^{\prime \prime}-80^{\prime \prime}$ which at redshifts $\sim 0.05$ are equivalent to linear resolutions of $44-80 \mathrm{kpc}$, sufficient to study individual galaxies; but at $z=1$ imply linear resolutions of $360-640 \mathrm{kpc}$ that are not sufficient to identify unique optical counterparts. The Faint Images of the Radio Sky at Twenty centimeters (FIRST,[5]) survey at $1.4 \mathrm{GHz}$ with a resolution of $\sim 5^{\prime \prime}$ is useful to resolve sources on linear scales of $\sim 50 \mathrm{kpc}$. However, FIRST does not cover the full sky and is relatively shallow $\left(\sim 0.2 \mathrm{mJy}^{\text {beam }}{ }^{-1}\right)$ to detect faint emission from galaxies and the ICM. Deep surveys sample limited regions of the sky and thus cannot be effectively used to study large samples of high redshift clusters. Thus pointed observations of a sample of galaxy clusters at high redshifts are needed in order to probe the radio sources they host.

The energy density of Cosmic Microwave Background (CMB) photons increases with redshift as $(1+z)^{4}$, and thus the inverse Compton (IC) losses are the main source of energy loss for the relativistic electrons responsible for the synchrotron emission at high redshifts. The equivalent IC field, $B_{I C}(\mu \mathrm{G}) \approx 3.2(1+z)^{2}$, is about $12.8 \mu \mathrm{G}$ at redshift 1 . Moreover due to the cosmological redshift, observations at $1.2 \mathrm{GHz}$ are sampling a rest frequency of $2.4 \mathrm{GHz}$ at the source. Thus to make a comparison with the spectra of nearby sources known around $1-1.4 \mathrm{GHz}$, observations of high redshift clusters at $0.5-0.7 \mathrm{GHz}$ are needed.

\section{Massive and Distant Clusters of the WISE Survey}

The MaDCoWS $[6,7]$ is a new IR-selected galaxy cluster survey based on the all-sky catalogues of the Wide-field Infrared Survey Explorer (WISE, [8]). The WISE infrared and Sloan Digital Sky Survey (SDSS) DR8 optical photometry [9] were combined to robustly isolate galaxy clusters at $\mathrm{z} \sim 1$ in the northern hemisphere. The selection through IR colour magnitude diagram allows to select high redshift galaxies.

High redshift clusters have been extracted earlier mainly through deep surveys of limited regions of the sky. The 10,000 $\mathrm{deg}^{2}$ survey footprint of WISE is four times larger than the South Pole Telescope - Sunyaev Ze'ldovich (SPT-SZ) Survey [10] and 1000 times larger than the area of the IRAC Distant Cluster Survey (IDCS), in which the most massive galaxy cluster at $z>1.5$ known to date was found [11, 12]. Given the unprecedented volume surveyed at high redshift, the MaDCoWS sample is expected to contain a large number of very massive and distant clusters [13]. There are a total of 19 spectroscopically confirmed galaxy clusters in the sample with redshifts in the range $0.7<z<1.3$.

The MaDCoWS clusters span a variety in their properties. Some of the MaDCoWS clusters are relaxed, with a centrally concentrated core of galaxies, a well-defined red sequence, and a few emission-line objects. But others span the range of possible combinations of these characteristics, 


\begin{tabular}{lcccrrr}
\hline Cluster & $\begin{array}{c}R A_{J 2000} \\
\mathrm{hh} \mathrm{mm} \mathrm{ss}\end{array}$ & $\begin{array}{c}D E C_{J 2000} \\
\mathrm{dd} \mathrm{mm} \mathrm{ss}\end{array}$ & $z$ & $\begin{array}{r}\mathrm{M}_{500} \\
10^{14} \mathrm{M}_{\odot}\end{array}$ & $\begin{array}{r}v_{o b s} \\
\mathrm{MHz}\end{array}$ & $\begin{array}{c}t_{\text {obs }} \\
\mathrm{min}\end{array}$ \\
\hline MOO_J0012+1602 & 001213.0 & +160216 & 0.944 & $1.4 \pm 0.5$ & 610 & 125 \\
& & & & & 1280 & 100 \\
MOO_J0133-1057 & 013355.6 & -105744 & 0.957 & & 610 & 125 \\
& & & & & 1280 & 175 \\
MOO_J0212-1813 & 021204.1 & -181413 & 1.098 & & 610 & 150 \\
MOO_J0224-0620 & 022451.3 & -062017 & 0.816 & & 610 & 175 \\
MOO_J1155+3901 & 115545.4 & +390106 & 1.009 & $2.9 \pm 0.7$ & 610 & 175 \\
MOO_J1514+1346 & 151443.8 & +134632 & 1.059 & $2.2 \pm 0.6$ & 610 & 150 \\
& & & & & 1280 & 150 \\
\hline
\end{tabular}

Table 1: Basic information of MaDCoWS clusters [13] with the GMRT observing frequencies, $v_{o b s}$ and duration $t_{\text {obs }}$.

some having a few emission-line objects but little spatial concentration in the core region, and others having a relatively high fraction of emission-line galaxies and a well-formed cluster core. Thus MaDCoWS is an unbiased starting point to study the properties of radio sources in high redshift galaxy clusters.

\subsection{Sample}

We extracted a sub-sample from the MaDCoWS based on the criterion of the occurrence of a radio source detected in the NVSS within $2^{\prime}$ of the cluster position. At the resolution of the NVSS the source cannot be uniquely identified with optical/infra-red detected galaxies. Among the selected sample, masses $\left(\mathrm{M}_{500}\right)$ are available for 3 clusters through Combined Array for Research in Millimetre-wave Astronomy (CARMA) detections [13] and are in the range $1.4-3 \times 10^{14} \mathrm{M}_{\odot}$ (Tab. 1).

\section{GMRT observations and data reduction}

Observations at $610 \mathrm{MHz}$ and L-band of five MaDCoWS clusters were carried out with the Giant Metrewave Radio Telescope (GMRT) in Cycle 28. The FITS data were processed with 'Yet Another Flagger' (N. Mohan, private communication) to remove radio frequency interference. The rest of the processing was carried out in AIPS. Additional manual flagging to remove bad antennas and time ranges was done. The data were then calibrated and imaged using the AIPS task 'imagr'. A few rounds of phase only self-calibration and a final round of amplitude and phase self-calibration were carried out.

\section{Results}

The radio images of the clusters MOO_J1155+3901, MOO_J0012+1602, MOO_J1514+1346 and MOO_J0133-1057 have been made (Tab. 2). Within $500 \mathrm{kpc}$ radius from the cluster centre 


\begin{tabular}{lccccc}
\hline Cluster & $\begin{array}{c}R A_{J 2000} \\
\text { hh mm ss }\end{array}$ & $\begin{array}{c}D E C_{J 2000} \\
\mathrm{dd} \mathrm{mm} \mathrm{ss}\end{array}$ & $\begin{array}{c}v_{o b s} \\
\mathrm{MHz}\end{array}$ & $\begin{array}{c}\mathrm{rms} \\
\mathrm{mJy} \mathrm{b}^{-1}\end{array}$ & $\begin{array}{c}\text { Beam } \\
\text { "×", p. a. }\end{array}$ \\
\hline MOO_J0012+1602 & 001213.0 & +160216 & 1280 & 0.045 & $2.6 \times 2.0,63.4^{\circ}$ \\
MOO_J0133-1057 & 013355.6 & -105744 & 610 & 0.051 & $5.1 \times 4.9,31.5^{\circ}$ \\
MOO_J1155+3901 & 115545.4 & +390106 & 610 & 0.04 & $5.4 \times 4.1,43.2^{\circ}$ \\
MOO_J1514+1346 & 151443.8 & +134632 & 610 & 0.06 & $5.0 \times 4.0,42.7^{\circ}$ \\
\hline
\end{tabular}

Table 2: GMRT images of the MaDCoWS clusters.

(MaDCoWS position), we looked for radio sources with identification in 3.6 micron WISE images. For example, the $610 \mathrm{MHz}$ image of MOO_J1514+1346 is shown in Fig. 1. Two radio souces near the cluster centre can be associated with two spectroscopically identified galaxies in this cluster. The third radio source within $500 \mathrm{kpc}$ radius, has a 3.6 micron counterpart but no associated spectroscopically identified galaxy.

The cluster MOO_J0012+1602 contains a double source at the centre for which no spectroscopically identified counterpart is present. In $610 \mathrm{MHz}$ images of MOO_J1155+3901 and MOO_J0133-1057, single radio sources are detected within $500 \mathrm{kpc}$ radius of each of the cluster centre. Analysis of the remaining data are ongoing.

\section{Discussion and conclusions}

Radio sources in galaxy clusters at high redshift are of interest to study the evolution of the mode of accretion of galaxies and the evolution of the cluster itself. At lower redshift clusters are known to contain radio sources associated with galaxies and also those associated with the ICM. We have presented preliminary results from our project to study radio sources in high redshift clusters $(z \sim 1)$ using the GMRT. Radio data analysis of three clusters at $610 \mathrm{MHz}$ and one cluster at 1280 $\mathrm{MHz}$ have been completed. In the analysis of these four clusters, we have not detected diffuse radio sources associated with the ICM. Within $500 \mathrm{kpc}$ radius from the cluster centre, we detect radio double sources or point sources. The identification of spectroscopically confirmed cluster member galaxies with radio sources is ongoing.

Relativistic electrons suffer dominant losses of energies via IC losses to CMB photons. Therefore detections of diffuse radio sources in clusters at these redshifts are a challenge. In the massive cluster 'El Gordo' at redshift of 0.87 a radio halo and relics have been detected [14]. With a mass of $M_{500}=(1.17 \pm 0.17) \times 10^{15} \mathrm{M}_{\odot}$, 'El Gordo' is about 3 - 5 times more massive than the MaDCoWS clusters that are surveyed. According to the studies carried out in the past decade, massive and merging clusters have been found more likely to host radio halos than less massive clusters $[15,16]$. However the less massive clusters at all redshifts have not been systematically surveyed and according to the predictions of the turbulent re-acceleration model [16] steep spectrum and less powerful radio halos may be present but have never been looked for. The non-detections so far of cluster-scale radio sources in MaDCoWS are thus not a surprise.

The other aspect of our study is to build the statistics of radio sources in high redshift galaxy clusters. The MaDCoWS sample is being followed up to identify cluster member galaxies using 


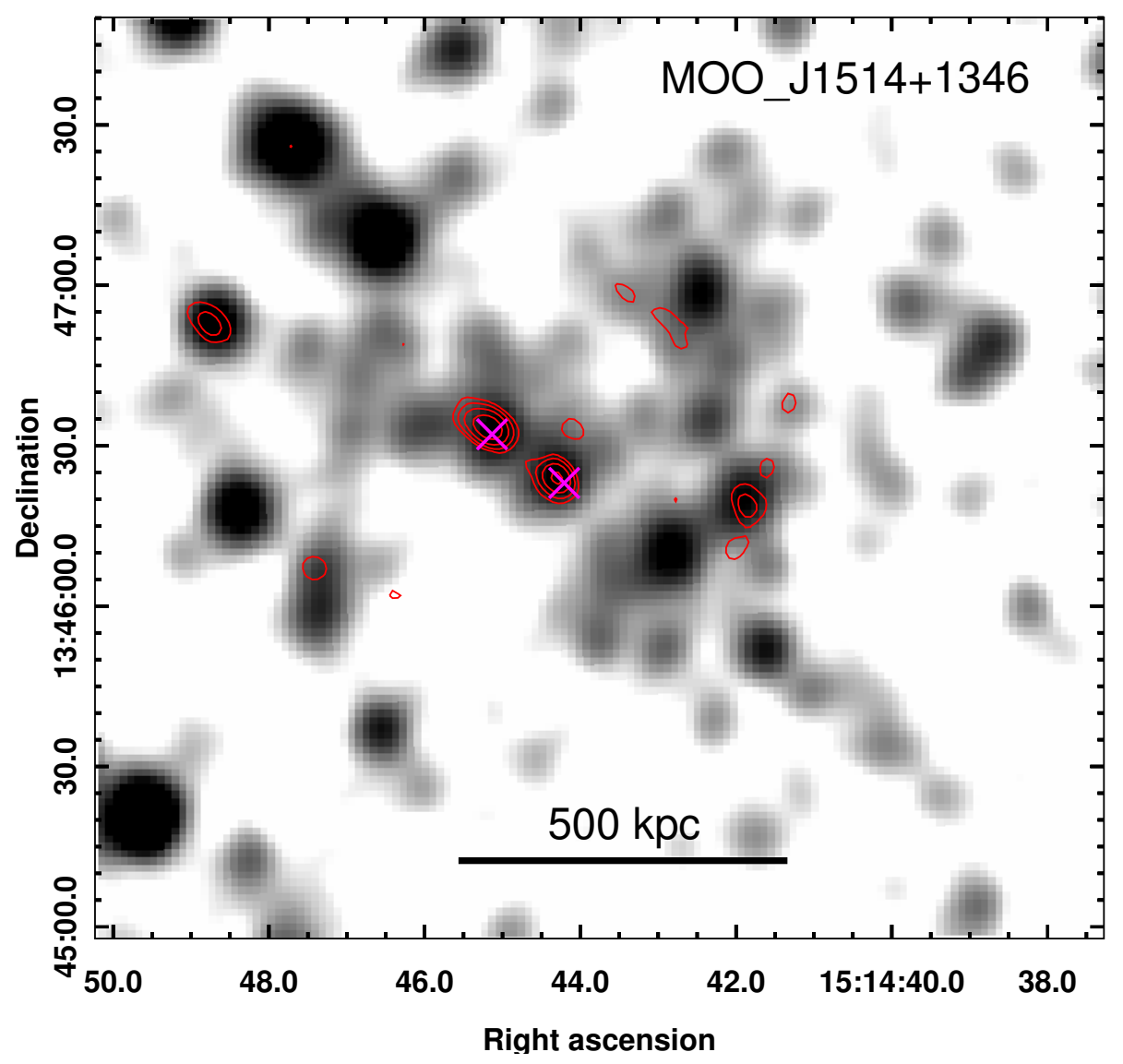

Figure 1: GMRT $610 \mathrm{MHz}$ contours (red) overlaid on the WISE 3.6 micron image of MOO_J1514+1346 shown in grey-scale. The contour levels are at $3 \sigma \times[ \pm 1,2,4, \ldots] \mathrm{mJy} \mathrm{b}^{-1}$ where $\sigma=0.06 \mathrm{mJy} \mathrm{b}^{-1}$ and the beam is $5^{\prime \prime} \times 4^{\prime \prime}$, p. a. $42.7^{\circ}$. The crosses mark the positions of two spectroscopically identified galaxies with IDs 1667 and 2668 from the left to the right [7].

spectroscopy [7]. Our study at 610 and $1280 \mathrm{MHz}$ with resolutions of $5^{\prime \prime}-2^{\prime \prime}$ will be able to identify radio sources with the galaxies (e.g. Fig. 1). The radio sources in MaDCoWS sample will be used to build a radio luminosity function to compare with that known at lower redshifts [17]. The analysis of GMRT data on the remaining clusters and further observations of more clusters will enable to build the sample of radio sources for this study.

\section{Acknowledgments}

RK thanks N. Mohan for pre-processing the data using the YAF. We thank the staff of the GMRT that made these observations possible. GMRT is run by the National Centre for Radio Astrophysics of the Tata Institute of Fundamental Research. This research has made use of the NASA/IPAC Extragalactic Database (NED) which is operated by the Jet Propulsion Laboratory, California Institute of Technology, under contract with the National Aeronautics and Space Administration. This publication makes use of data products from the Wide-field Infrared Survey Explorer, which is a joint project of the University of California, Los Angeles, and the Jet Propul- 
sion Laboratory/California Institute of Technology, funded by the National Aeronautics and Space Administration.

\section{References}

[1] J. J. Condon, W. D. Cotton, E. W. Greisen, Q. F. Yin, R. A. Perley, G. B. Taylor et al., The NRAO VLA Sky Survey, AJ 115 (1998) 1693-1716.

[2] T. Mauch, T. Murphy, H. J. Buttery, J. Curran, R. W. Hunstead, B. Piestrzynski et al., SUMSS: $a$ wide-field radio imaging survey of the southern sky - II. The source catalogue, MNRAS 342 (2003) 1117-1130, [astro-ph/0303188].

[3] W. M. Lane, W. D. Cotton, S. van Velzen, T. E. Clarke, N. E. Kassim, J. F. Helmboldt et al., The Very Large Array Low-frequency Sky Survey Redux (VLSSr), MNRAS 440 (2014) 327-338, [1 404.0694 ].

[4] R. B. Rengelink, Y. Tang, A. G. de Bruyn, G. K. Miley, M. N. Bremer, H. J. A. Roettgering et al., The Westerbork Northern Sky Survey (WENSS), I. A 570 square degree Mini-Survey around the North Ecliptic Pole, A\&ApS 124 (1997).

[5] R. H. Becker, R. L. White and D. J. Helfand, The FIRST Survey: Faint Images of the Radio Sky at Twenty Centimeters, ApJ 450 (1995) 559.

[6] D. P. Gettings, A. H. Gonzalez, S. A. Stanford, P. R. M. Eisenhardt, M. Brodwin, C. Mancone et al., The Massive Distant Clusters of WISE Survey: The First Distant Galaxy Cluster Discovered by WISE, ApJL 759 (2012) L23, [1205 . 7092$].$

[7] S. A. Stanford, A. H. Gonzalez, M. Brodwin, D. P. Gettings, P. R. M. Eisenhardt, D. Stern et al., The Massive and Distant Clusters of WISE Survey. II. Initial Spectroscopic Confirmation of $z \sim 1$ Galaxy Clusters Selected from 10,000 deg ${ }^{2}$, ApJS 213 (2014) 25, [1403.3361].

[8] E. L. Wright, P. R. M. Eisenhardt, A. K. Mainzer, M. E. Ressler, R. M. Cutri, T. Jarrett et al., The Wide-field Infrared Survey Explorer (WISE): Mission Description and Initial On-orbit Performance, AJ 140 (2010) 1868-1881, [1008.0031].

[9] H. Aihara, C. Allende Prieto, D. An, S. F. Anderson, É. Aubourg, E. Balbinot et al., The Eighth Data Release of the Sloan Digital Sky Survey: First Data from SDSS-III, ApJS 193 (2011) 29, [1101.1559].

[10] L. E. Bleem, B. Stalder, T. de Haan, K. A. Aird, S. W. Allen, D. E. Applegate et al., Galaxy Clusters Discovered via the Sunyaev-Zel'dovich Effect in the 2500-Square-Degree SPT-SZ Survey, ApJS 216 (2015) 27, [1409.0850].

[11] M. Brodwin, A. H. Gonzalez, S. A. Stanford, T. Plagge, D. P. Marrone, J. E. Carlstrom et al., IDCS J1426.5+3508: Sunyaev-Zel'dovich Measurement of a Massive Infrared-selected Cluster at $z=1.75$, ApJ 753 (2012) 162, [1205.3787].

[12] A. H. Gonzalez, S. A. Stanford, M. Brodwin, C. Fedeli, A. Dey, P. R. M. Eisenhardt et al., IDCS J1426.5+3508: Cosmological Implications of a Massive, Strong Lensing Cluster at z = 1.75, ApJ 753 (2012) 163, [1205.3788].

[13] M. Brodwin, C. H. Greer, E. M. Leitch, S. A. Stanford, A. H. Gonzalez, D. P. Gettings et al., The Massive and Distant Clusters of WISE Survey. III. Sunyaev-Zel'dovich Masses of Galaxy Clusters at $z \sim 1$, ApJ 806 (2015) 26, [1410.2355].

[14] R. R. Lindner, A. J. Baker, J. P. Hughes, N. Battaglia, N. Gupta, K. Knowles et al., The Radio Relics and Halo of El Gordo, a Massive z = 0.870 Cluster Merger, ApJ 786 (2014) 49, [1310 . 678 6]. 
[15] R. Cassano, S. Ettori, G. Brunetti, S. Giacintucci, G. W. Pratt, T. Venturi et al., Revisiting Scaling Relations for Giant Radio Halos in Galaxy Clusters, ApJ 777 (2013) 141, [1306.4379].

[16] G. Brunetti and T. W. Jones, Cosmic Rays in Galaxy Clusters and Their Nonthermal Emission, International Journal of Modern Physics D 23 (Mar., 2014) 30007, [1401. 7519].

[17] M. Branchesi, I. M. Gioia, C. Fanti, R. Fanti and R. Perley, The radio luminosity function of the NEP distant cluster radio galaxies, A\&A 446 (2006) 97-111, [astro-ph/ 0509138 ]. 